\title{
Nordiques
}

33 | 2017

La transition des villes nordiques : quelles innovations territoriales en périphérie?

\section{Kirkenes, Nikel et leurs environs explorés par le projet " Dark Ecology » : une excursion en dehors de la zone de confort}

Jørn Riseth

\section{(2) OpenEdition \\ Journals}

\section{Édition électronique}

URL : https://journals.openedition.org/nordiques/3225

DOI : 10.4000 /nordiques.3225

ISSN : 2777-8479

Éditeur :

Association Norden, Bibliothèque de Caen la mer

Édition imprimée

Date de publication : 1 mai 2017

Pagination : 79-90

ISBN : 978-2-9544654-9-4

ISSN : $1761-7677$

\section{Référence électronique}

Jørn Riseth, « Kirkenes, Nikel et leurs environs explorés par le projet « Dark Ecology » : une excursion en dehors de la zone de confort », Nordiques [En ligne], 33 | 2017, mis en ligne le 14 janvier 2022 consulté le 02 février 2022. URL : http://journals.openedition.org/nordiques/3225 ; DOI : https:// doi.org/10.4000/nordiques.3225 


\section{Kirkenes, Nikel et leurs environs explorés par le projet « Dark Ecology » une excursion en dehors de la zone de confort}

Jørn Riseth*

\section{RÉSUMÉ}

Situées le long des côtes arctiques de la mer de Barents, les villes industrielles de Kirkenes et Nikel sont dépourvues de structures pour exposer des ouvres d'art contemporain. Le projet artistique "Dark Ecology " a choisi cette zone frontalière entre la Norvège et la Russie pour réfléchir aux problèmes environnementaux. Des artistes appartenant au courant "l'art des nouveaux médias " ont été invités à explorer cette région de l'Arctique et à créer des œuvres à partir d'un nouveau contexte. L'article vise à montrer comment les artistes ont tenté de reformuler les concepts de nature et d'environnement en faisant de l'expérience artistique un instrument permettant de voir les problèmes environnementaux de notre époque sous un autre angle.

\section{ABSTRACT}

The industrial cities of Kirkenes and Nikel, in the arctic region of the Barents Sea, both lack structures for exposing contemporary art. The art project "Dark Ecology" chose this border area between Norway and Russia to reflect on environmental problems. Artists belonging to the movement called "new media art" were invited to test their ideas on ecology in a new context. This article aims to show how the artists tried to reformulate the concepts of nature and environment using the artistic experience as an instrument which allows perceiving environmental problems from a new perspective.

* Jørn Riseth est maitre de langue de norvégien au Département d'études nordiques de l'université de Caen Normandie et doctorant à l'école doctorale 558 "Histoire, Mémoire, Patrimoine, Langage " à l'université de Caen Normandie. Il prépare une thèse en littérature norvégienne sous la direction de Harri Veivo. 
Les villes de Kirkenes et de Nikel se trouvent à 54 kilomètres l'une de l'autre, de part et d'autre de la frontière entre la Norvège et la Russie. Le projet « Dark Ecology " a choisi ces deux villes voisines et leurs alentours pour y déployer une série de manifestations artistiques entre 2014 et 2016. À la fois touchée par la pollution locale et très exposée aux conséquences des changements climatiques globaux, la région de la mer de Barents constitue un cas particulièrement intéressant d'un point de vue écologique. Afin d'entamer une discussion sur les relations entre l'Homme et son environnement, les initiateurs du projet «Dark Ecology » ont proposé aux artistes des résidences en Arctique leur permettant de créer des œuvres sur place. La démarche originale du projet a été d’organiser des voyages dans la région frontalière entre la Norvège et la Russie au cours desquels artistes, chercheurs et commissaires d'exposition ont pu se côtoyer. En proposant aux participants d'assister à la présentation d’œuvres commissionnées, performances ou conférences, le projet a favorisé la création de réseaux entre le monde artistique et le monde scientifique. Ces excursions ont également été l’occasion de rencontrer la population locale. Par le fait d’avoir été emmenés dans des paysages ravagés par les activités industrielles, les participants ont été obligés de quitter leurs arènes habituelles pour saventurer hors de leur zone de confort.

Cet article commentera quelques-unes de ces œuvres pour montrer la diversité qui marque l'approche des questions environnementales. Il s'intéresse pour cela à la façon dont le projet a su créer des échanges autour des problèmes écologiques auxquels la population de cette région est confrontée. Au-delà, il s'est donné pour objectif d'explorer la façon dont les arts peuvent contribuer à comprendre l'expérience de la vie à une époque, la nôtre, où les activités humaines transforment les écosystèmes à tous les niveaux.

\section{LE CONTEXTE ENVIRONNEMENTAL}

Il est nécessaire au préalable de faire une brève description du contexte environnemental de cette zone frontalière. L'histoire de Kirkenes et celle de Nikel sont toutes deux marquées par l'exploitation de ressources minérales. Comme son nom l'indique, la ville de Nikel est productrice de nickel, et une fonderie se trouve près du centre-ville. Cette usine et une autre produisant des pellets de nickel à Zapoljarnij (à 30 kilomètres de là) émettent de grandes quantités de dioxyde de soufre $\left(\mathrm{SO}_{2}\right)$ et de métaux lourds. Selon l'autorité norvégienne de la recherche sur l'air (NILU), les émissions de $\mathrm{SO}_{2}$ sont réduites depuis trente ans, mais elles représentaient encore en 2014 cinq fois la quantité de $\mathrm{SO}_{2}$ émise dans toute la Norvège. Les mesures du NILU montrent qu'à certains endroits près de la frontière, les limites maximales de concentration de $\mathrm{SO}_{2}$ dans l'air fixées par les autorités norvégiennes ont été dépassées chaque année depuis 2010. En 
ce qui concerne les émissions de métaux lourds (le nickel, le cuivre, le cobalt et l'arsenic), il y a eu une nette augmentation depuis 2004. Les mesures prises du côté norvégien sur la concentration des métaux lourds ne correspondent pas aux chiffres donnés par les autorités russes ${ }^{1}$. Les émissions de $\mathrm{SO}_{2}$ et de métaux lourds détruisent la végétation, contaminent les lacs et le sol des deux côtés de la frontière entre la Norvège et la Russie.

Dans la commune de Syd-Varanger, dont Kirkenes est le chef-lieu, se trouvent les gisements de minerai de fer les plus riches de Norvège, et une mine à ciel ouvert à Bjørnevatn a été exploitée jusqu'en 2015. La transformation du minerai en boulettes de fer aussi bien que l'expédition par bateau se font à Kirkenes. Le fractionnement du minerai du fer par l'eau a produit des résidus qui polluent le fjord en face de Kirkenes, et la fonderie qui transforme le fer en pellets a causé une dispersion de dioxines par l'air. La consommation du poisson de plusieurs lacs à proximité de Kirkenes est déconseillée en raison des concentrations de dioxine trop élevées ${ }^{2}$. La mine de fer à ciel ouvert de Bjørnevatn, qui est abandonnée depuis la faillite de la société Sydvaranger Gruve, reste comme une plaie béante dans le paysage.

Les effets néfastes de l'exploitation de minerais sont évidents dans les deux villes. Des problèmes de pollution liés à l'extraction de métaux menacent la faune, la flore, ainsi que la santé humaine. À quoi il faut ajouter que les paysages industriels de la région de Barents sont voisins d'une nature encore peu exploitée par l'Homme. Le parc national d'Øvre Pasvik qui se trouve au sud de la commune de Syd-Varanger, est connu pour ses vieilles forêts de pins et la plus grande population d'ours bruns de Norvège.

Les effets des changements climatiques globaux s'ajoutent à ces problèmes locaux de pollution. Cependant, pour les habitants de la région, le réchauffement du climat est à la fois une source d'inquiétude et l'origine d'un certain optimisme. La fonte de la banquise dans la mer de Barents permettrait d'exploiter de nouveaux gisements de pétrole et de gaz. Les changements climatiques pourraient aussi donner accès à de nouvelles ressources de pêche. La hausse des températures a en outre rendu possible l'ouverture de la route maritime du Nord-Est à la navigation, ce qui permet de développer les relations commerciales avec l'Asie. Dans les années à venir, les habitants de la région de Barents connaîtront peut-être un essor économique sans précédent. Lors de la conférence Arctic Frontiers à Tromsø en 2013, le ministre norvégien des Affaires étrangères, Espen Barth Eide, a affirmé à

1 Données publiées par NILU le 13 février 2014, [http://www.nilu.no/Nyhetsarkiv/tabid/74/ language/nb-NO/NewsId/619/Fortsatt-hye-konsentrasjoner-av-miljgifter-i-SrVaranger-grunnet-utslippfra-Russland.aspx] (consulté le 30 mars 2017).

2 Article du journal I Finnmark du 7 septembre 2010, [http://www.ifinnmark.no/nyheter/giftig-fiski-kirkenes/s/1-30002-5407527] (consulté le 30 mars 2017). 
propos de la nouvelle situation dans l'Arctique que « les défis liés au changement climatique augmentent, mais les opportunités aussi, l'essentiel étant de maintenir un équilibre entre les deux $\|^{3}$. En Norvège comme en Russie, ces perspectives ont de quoi faire rêver les compagnies pétrolières et trembler d'impatience ceux qui souhaitent développer les transports et le commerce avec l'Asie.

\section{LE PROJET « DARK ECOlogY » ET SES PARTENAIRES}

Le projet «Dark Ecology » a été initié par Hilde Methi, une commissaire d'exposition basée à Kirkenes, et Sonic Acts, une plate-forme hollandaise qui travaille à l'intersection entre arts, science et théorie. Le projet a été organisé en coopération avec différents partenaires en Norvège et en Russie. Il a aussi obtenu le soutien de l'Union européenne. Le financement a pour une grande partie été pris en charge par l'organisme norvégien Barentskult, qui institue une coopération entre le ministère des Affaires étrangères, le ministère de la Culture et les administrations régionales du Finnmark, du Troms et du Nordland. Cet organisme a pour objectif de renforcer les contacts culturels entre la Norvège et la Russie dans le Nord.

L'un des initiateurs de Sonic Arts, Arie Altena, raconte que l'idée du projet "Dark Ecology » a pris forme lors d'un voyage dans la région en 2012. En visite à Nikel, il a pu observer la fumée chargée de pollution qui sortait des cheminées des usines pour se poser sur la neige autour de la ville. Il a été stupéfait par le paysage industriel qui avoisinait la belle nature de la vallée de Pasvik. La complexité évidente des problèmes environnementaux l'a amené à choisir la région et le contexte de Kirkenes et de Nikel pour réfléchir aux connections intimes entre pollution, métaux, industrie et nature ${ }^{4}$.

\section{LES CONCEPTS DE NATURE ET D'ENVIRONNEMENT}

Le projet "Dark Ecology " doit son nom au philosophe Timothy Morton. Dans son ouvrage Ecology without Nature. Rethinking Environmental Aesthetics (2007), Morton propose la notion de dark ecology pour désigner une conscience écologique qui prend acte du fait que la catastrophe écologique a déjà eu lieus. Nous sommes entrés dans l'époque de l'anthropocène, une nouvelle ère géologique où l'impact humain sur l'environnement est si important qu'il n'est plus

3 Anne Denis, "La conquête glaciale », Libération, 3 février 2013, [http://www.liberation.fr/ futurs/2013/02/03/arctique-la-conquete-glaciale_878977] (consulté le 30 mars 2017).

4 Mirna Belina, "Dark Ecology. Outside the Comfort Zone ", in Living Earth. Field Notes from the Dark Ecology Project 2014-2016, M. Belina (éd.), Amsterdam, Sonic Acts Press, 2016, p. 11.

5 Timothy Morton, Ecology without Nature. Rethinking Environmental Aesthetics, Cambridge, Harvard University Press, 2007. 
possible de maintenir la distinction entre l'Homme et la nature ${ }^{6}$. Pour vivre à cette époque, il faut s'habituer à une réalité étrange et sombre, notamment celle de savoir que les conséquences de l'activité humaine sur l'environnement sont irréversibles et d'une telle ampleur que l'on peut difficilement prendre de la distance par rapport aux problèmes écologiques. Faisant partie du mouvement de la "philosophie de l'objet " (object-oriented philosophy), Morton récuse la vision anthropocentriste du monde. Il défend l'idée que les objets existent indépendamment de la perception humaine. L'Homme n'est pas une espèce qui peut se mettre à l'écart, il fait partie d'un réseau où tous les êtres, vivants et non vivants, existent dans une communauté de destin. Morton propose la notion de mesh pour désigner ce réseau où les éléments sont interconnectés. La pensée écologique ne devrait pas privilégier un genre d'être de ce réseau au détriment d'un autre, selon Morton. Participant au premier voyage organisé par " Dark Ecology ", Morton s'est rendu à Nikel. Suite à sa visite, il a exprimé qu'il était d'abord triste de voir la forêt dévastée par la pollution de l'usine de nickel et la ville pleine d'objets cassés. Mais, malgré la présence très explicite de la mort, il a trouvé une chaleur et une sorte de joie dans cette ville. Morton estime que le seul côté positif de la réalité environnementale est à trouver dans une reconnaissance de la situation, et que c'est là précisément que l'art pourrait jouer un rôle. Il admet que la situation est sombre, mais il considère que l'expression artistique peut nous amener à trouver des manières de vivre à l'époque de l'anthropocène $e^{7}$. Dans une intervention lors du premier voyage du projet "Dark Ecology », Timothy Morton a reproché au courant de «l'écologie profonde » de défendre une vision nostalgique de la nature. Le courant visé par cette critique a été fondé par le philosophe norvégien Arne Næss (1912-2009). Dans un ouvrage intitulé Økologi, samfunn og livsstil (1974), Næss a développé le concept de l'écologie profonde. Ce livre est paru en français en 2013 sous le titre Écologie, communauté et style de vie ${ }^{8}$. Pour Næss, l'idée que chaque être vivant a sa valeur intrinsèque est intuitive. Comme Morton, il pense qu'il est nécessaire de remplacer l'approche anthropocentriste de l'écologie et de reconnaître qu'il existe une interdépendance entre tous les êtres vivants, l'homme y compris. Il s'oppose à ce qu'il appelle l'écologie superficielle, c'est-àdire à la vision que l'homme serait capable de remédier aux problèmes environnementaux à l'aide de la technologie. Malgré des points en commun, Morton prend ses distances avec l'écologie profonde en estimant que celle-ci représente une idéalisation de la nature. Selon Morton, la nostalgie d'une harmonie perdue entre l'Homme et la nature nous empêche de développer une véritable pensée

6 Pour une introduction à la notion de l'anthropocène, voir l'article de Jørgen Bruhn dans la revue Ekfrase, $1^{\text {er }}$ février 2016, [https:/www.idunn.no/ekfrase/2016/01-02/introduction_to_the_ anthropocene_theme] (consulté le 30 mars 2017).

7 Timothy Morton, "What Is Dark Ecology? ", in Living Earth. Field Notes from the Dark Ecology Project 2014-2016, op. cit., p. 30.

8 Arne Næss, Écologie, communauté et style de vie, Bellevaux, Éditions Dehors, 2013. 
écologique. Il pense qu'on ne pourra jamais retrouver une nature intacte, comme elle a dû exister avant l'industrialisation.

Les initiateurs du projet "Dark Ecology » se sont joints aux idées de Morton en affirmant qu'il était nécessaire de se débarrasser de la notion de nature. L'affirmation suivante, publiée sur le site du projet, résume leur position : "Ecology does not privilege the human, it is not something beautiful, and it has no real use for the old concept of Nature. " Ils pensent qu'il faut abandonner l'idée selon laquelle l'écologie est intrinsèquement salutaire, et ils prennent leurs distances avec la notion traditionnelle de la nature défendue par le mouvement de l'écologie profonde.

Parmi les invités au deuxième voyage du projet " Dark Ecology " en 2015, figurait le philosophe Graham Harman, lui aussi membre du courant de la "philosophie de l'objet ». Son travail sur l'outil cherche à identifier des relations interobjectales qui échappent au contrôle de l'Homme. Dans une anthologie publiée à la fin du projet "Dark Ecology ", Harman discute la notion de l'environnement telle qu'elle a été formulée par le biologiste Jacob von Uexküll1 ${ }^{10}$. Dans un ouvrage devenu classique, Milieu animal et milieu humain, von Uexküll raconte la vie passionnante de la bruche du pois, un insecte coléoptère appartenant à la grande famille des chrysomèles, dont les larves se nourrissent de graines de pois et de haricots. Quand la chair du pois est encore tendre, la larve creuse un canal jusqu’à la surface de la graine. Mais ce canal ne servira qu'au moment où l'insecte sera adulte. La bruche du pois pourra alors sortir du pois dont la chair aura durci. Il ne semble pas y avoir de signal perceptible dans l'environnement de l'insecte pour indiquer à la larve la nécessité d'une sortie de secours ${ }^{11}$. Selon von Uexküll, le signal n'existe pas dans l'environnement, mais a dû être ajouté par l'animal lui-même. Pour qualifier ce qui dépasse la structure perceptuelle de l'environnement, von Uexküll utilise la notion de magic. Graham Harman se déclare prêt à accepter le mot magic pour parler des cas où un humain ou un animal semblent agir au-delà du monde qu'ils sont capables de percevoir, même si la magie a une mauvaise réputation à l'époque moderne. Dans la théorie de von Uexküll, ces sauts " magiques " sont cruciaux, car sans eux les hommes et les animaux seraient enfermés dans leurs bulles respectives. Mais Harman rejette la supposition par van Uexküll que cette transgression soit rendue possible grâce aux projections subjectives ou à l'héritage de mystérieux schémas des ancêtres. La question est, dit-il, de savoir ce qu'est en réalité la magie. Pour parler de la façon dont un être est capable de bouger à l'extérieur de son environnement, il propose

9 Page web du «Dark Ecology Project ", [http://www.darkecology.net/about] (consulté le 30 mars 2017).

10 Graham Harman, "Magic Uexküll ", in Living Earth. Field Notes from the Dark Ecology Project 2014-2016, op. cit., p. 115.

11 Jakob von Uexküll, Milieu animal et milieu humain, Paris, Payot \& Rivages, 2010, p. 147-149. 
à la place la notion de l'esthétique. Selon Harman, la seule manière de comprendre pourquoi la larve creuse un tunnel dans le pois, c'est l'esthétique :

Even the larva does not just experience the pea as a bundle of present qualities, but a mysterious space of possibilities that forms a surplus beyond its current aspect. ${ }^{12}$

Harman fait donc une distinction entre la connaissance d'un objet et l'appréciation esthétique de celui-ci. Il soutient l'idée selon laquelle on peut décrire une œuvre artistique en listant toutes ses caractéristiques déterminées par des faits, mais l'objet en question reste malgré ces connaissances un mystère pour nous.

\section{CEUVReS COMMISSIONNÉES}

Les œuvres présentées par le projet "Dark Ecology » appartiennent au courant artistique souvent nommé " les arts des nouveaux médias ", réunissant l'art cinétique, l'art conceptuel, happening and performance et le Land Art. Sonic Acts s'est depuis longtemps intéressé aux projets artistiques qui répondent à leur localisation, c'est-à-dire à des œuvres qui problématisent le contexte environnemental, social et politique, d'un endroit spécifique. C'est dans cet esprit que le projet " Dark Ecology » a sollicité les artistes pour qu'ils aillent à l'extérieur explorer des paysages naturels et industriels de la zone frontalière entre la Norvège et la Russie. Les artistes ont répondu à cette invitation en créant des œuvres à partir d'éléments sonores, visuels ou matériels collectionnés dans la région ${ }^{13}$.

À la demande du projet " Dark Ecology », l'artiste néerlandais Joris Strijbos a créé une œuvre installée en plein air à Langøra, une colline à proximité de Kirkenes. L'endroit situé en pleine nature est tout de même visible de la ville. Travaillant dans une tradition kinesthésique, l'artiste a présenté une œuvre audiovisuelle faite à partir des mouvements du vent captés sur le site même. L'œuvre est constituée d'une quinzaine d'appareils identiques hauts de trois mètres, ressemblant à des robots. Interviewé par le journal local, Sør-Varanger Avis, l'artiste a expliqué avoir voulu créer une colonie de vie artificielle où chaque élément interagissait avec les autres. Il a comparé cette colonie avec des oiseaux volant en groupe ; un seul oiseau n'est pas capable de créer les mêmes motifs sonores et visuels qu'un groupe ${ }^{14}$. L'installation de Joris Strijbos répond à l'ambition du projet « Dark Ecology » de remettre en question l'idée traditionnelle de nature. En montrant que les robots interagissent et transforment des éléments sonores qui existent dans l'environnement, l'œuvre est une belle illustration de l'idée mortonienne selon laquelle la nature est un réseau cohérent englobant à la fois des êtres vivants et non vivants.

12 Graham Harman, op. cit., p. 130.

13 Mirna Belina, op. cit., p. 10.

14 Article de Birgitte Wisur Olsen, "En koloni av kunstig liv på byens tak », Sør-Varanger Avis, 25 août 2015, [http://sva.no/reportasjer/en-koloni-av-kunstig-liv-pa-byens-tak/19.4339] (consulté le 30 mars 2017). 


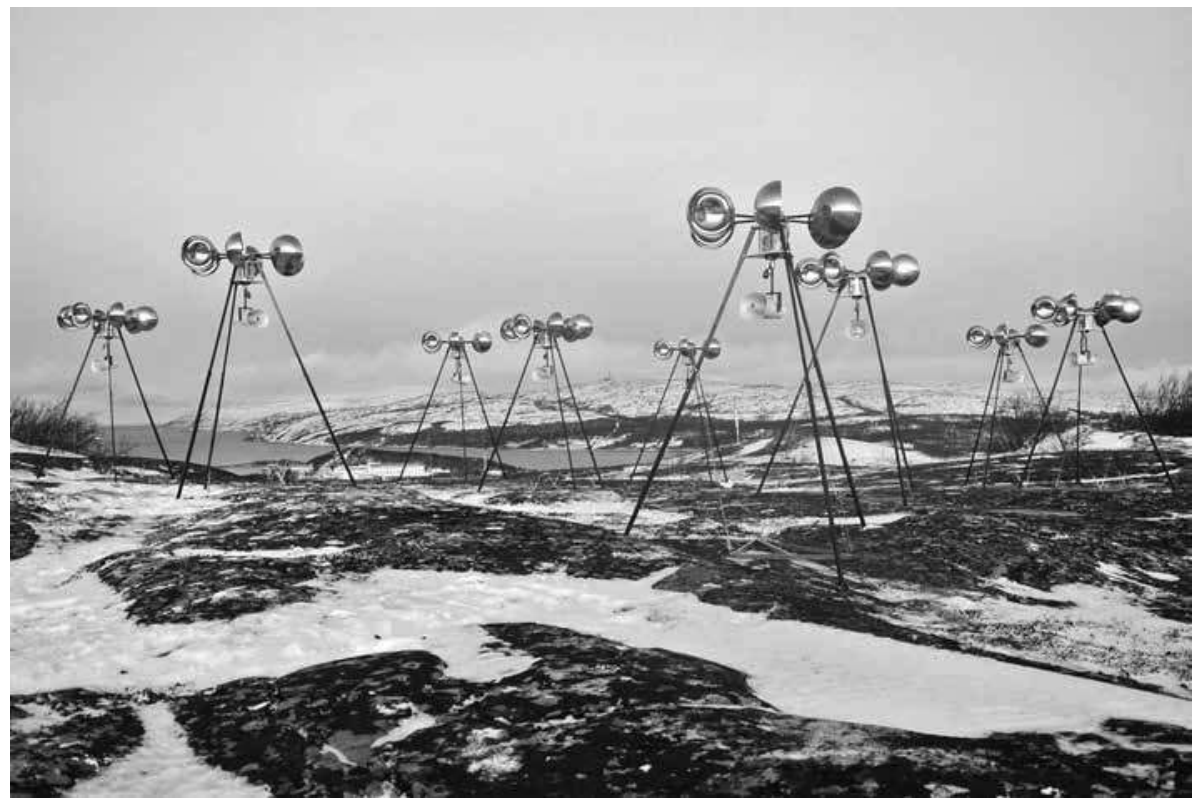

L’œuvre IsoScope de Joris Strijbos installée à Langøra, Kirkenes.

Photo : Dark Ecology

L'œuvre Nikel. The City as Material a été présentée par les deux architectes russes Tatjana Gorbachewskaja et Katya Larina. Travaillant dans un cadre défini par le courant philosophique New Materialism, leur ambition était de repenser les relations entre sujet et objet aussi bien qu'entre l'Homme et la nature ${ }^{15}$. La méthode qu'elles ont appliquée consiste à déplacer la focalisation de l'expérience humaine vers le matériel et aussi à reconnaître l'influence des forces non humaines pour expliquer des procès immatériels. Le nom de la ville de Nikel indique déjà la prééminence du matériel, et toute la vie sociale de cette ville a été déterminée en fonction de l'activité minéralière. Grâce à l'extraction du précieux nickel, les habitants de la ville ont profité de longues vacances, de retraites élevées et de structures sportives modernes. À l'époque de l'Union soviétique, la ville de Nikel avait des infrastructures mises en place et entretenues par l'État, mais à la chute du communisme, ce système artificiel s'est effondré, et les infrastructures sont en train de se dégrader. En étudiant la ville comme un système vivant, les architectes ont documenté sa dégradation matérielle par une série de photos. À Nikel, les matériaux se détériorent rapidement en raison des conditions météorologiques très rudes. Mais, soulignent les architectes, personne ne contrôle ce processus. Elles considèrent que la ville est un système écologique ayant sa propre logique. Leur idée de ville comme système écologique autorégulé est intrigante.

15 Mirna Belina, op. cit., p. 98-106. 
Premièrement, elle interroge la conception selon laquelle la nature est ce qui se trouve en dehors de la ville, en opposition à ce que l'homme a créé, défini comme la culture. Deuxièmement, elle inscrit dans une œuvre artistique la façon dont les matériaux de la ville ont une dynamique qui échappe au contrôle humain. Selon les architectes, les habitants de la ville ont réservé un accueil positif au projet. Étant donné que le développement de la ville depuis la chute du communisme représente pour beaucoup d'habitants une tragédie personnelle, les architectes ont été surprises par l'accueil favorable qu'elles ont rencontré.

L'artiste Margrethe Pettersen s'est intéressée à l'environnement sonore dans l'Arctique. Dans les environs de Kirkenes, elle a organisé une balade sonore, un soundwalk, sur un lac glacé lors du second voyage du projet « Dark Ecology » en novembre 2015. L'œuvre de Pettersen, Living Land. Below as Above, cherche à faire entendre des voix inaudibles. Pendant l'hiver arctique, toute la vie se trouve cachée ; une couverture de neige et de glace empêche le contact avec les espèces qui hibernent. Au cours des balades, les participants ont écouté une multitude de voix dont une plante au repos qui est intervenue à la première personne depuis le fond du lac:

Je suis une plante aquatique - l'utriculaire commune, Utricularia vulgaris - je flotte librement sans racine. Pendant les journées froides et sombres, je quitte la surface et, en forme de boule, je tombe au fond du lac - que j'appelle ma maison. C'est bien d'économiser de l'énergie, tu devrais essayer - au moins de réduire ta vitesse. ${ }^{16}$

Quand le narrateur s'imagine une plante qui parle, les frontières entre le monde végétal et le monde humain se trouvent effacées ; la dichotomie traditionnelle entre l'humain et les autres êtres est remplacée par une vision animiste du monde. L'attribution à une plante d'une subjectivité implique l'abandon de la perspective anthropocentriste. En utilisant le point de vue de la plante, le narrateur arrive à nous révéler ce que nous sommes incapables de comprendre nous-mêmes. Il nous confronte à un regard extérieur sur notre façon de faire avec l'environnement, et ce point de vue permet de formuler une critique à laquelle il est difficile d'échapper. Quand la plante nous conseille de faire comme elle, de se mettre en état d'hibernation au fond d'un lac pour économiser de l'énergie, l'originalité de la proposition est telle que cela nous intrigue. Pendant les soundwalks, l'artiste a également fait entendre des contes traditionnels Samis, minorité ethnique dont la voix a été inaudible pendant des siècles. Leur façon de vivre avec la nature se distingue du discours dominant qui a toujours prôné la prédation comme approche face aux paysages et aux animaux. L'œuvre est une proposition d'écouter d'autres voix que celles que nous connaissons déjà, et aussi de respecter d'autres manières

16 Britt Kramvik, Margrethe Pettersen, "Living Land : Below and Above ", in Living Earth. Field Notes from the Dark Ecology Project 2014-2016, op. cit., p. 136. C'est nous qui traduisons. 
de vivre avec la nature. L'artiste explique son point de vue dans un texte publié par le projet "Dark Ecology " où elle affirme que les différents modes de vie dans ces paysages ne devraient pas être perçus comme des conflits d'intérêt, mais plutôt comme des conflits ontologiques. Elle pense que les ontologies arctiques comme celle des Samis pourraient nous apprendre à établir d'autres relations avec l'environnement.

L'œuvre de Margrethe Pettersen met aussi en question la tendance à vouloir réduire des mondes multiples à un seul. Que le monde se présente différemment selon les capacités de perception dont on dispose est une idée que l'on peut retrouver chez le biologiste Jacob von Uexküll. Dans son ouvrage de 1909, Milieu animal et milieu humain, il explique que chaque animal, l'homme y compris, habite le monde d'une manière très subjective et que cette subjectivité est liée aux capacités sensorielles propres à chaque espèce ${ }^{17}$. Von Uexküll introduit la notion d'Umwelt selon laquelle chaque espèce dispose d'un univers qui lui est propre. Il est par exemple bien connu que les chiens ont des capacités sensorielles très différentes de celles de l'homme. Ayant un odorat très développé, les chiens s'intéressent aux passages d'autres animaux qui ont laissé des traces en forme de marquage urinaire. Même s'ils partagent un espace avec les humains, on peut supposer que les chiens se font des représentations des environs très différentes de celles des hommes ; les repères ne sont pas les mêmes. Cet exemple illustre l'idée de von Uexküll de l'existence de multiples environnements existant côte à côte. Mais même si les humains savent que les capacités de perception varient selon les espèces, nous avons du mal à admettre que d'autres êtres vivants puissent vivre ou voir autre chose que nous. Pour sortir de notre bulle autoréférentielle, Margrethe Pettersen propose de voir, de sentir et d'écouter autrement :

Respirez. Perturbez la vitesse. Détruisez la conception d'une seule chronologie.

Sentez. Écoutez. Réfléchissez, et travaillez dur pour raconter d'autres histoires. ${ }^{18}$

Les références à la vitesse et à la chronologie dans cette déclaration font penser aux exigences d'efficacité et de logique de notre époque. Le discours tenu par l'artiste est une invitation à dépasser les contraintes de la société moderne pour établir de nouvelles relations avec l'environnement.

L'installation sonore créée par Raviv Ganchrow est un autre exemple d'œuvre qui met en question nos capacités de perception. L'œuvre intitulée Long Wave Synthesis a été présentée dans le cadre du projet "Dark Ecology" en 2014 ${ }^{19}$. Réalisée près de l'aéroport de Kirkenes, l'installation sonore crée des ondes acoustiques en dessous du seuil de l'ouïe humaine. Les ondes longues interagissent avec

17 Jakob von Uexküll, op. cit.,

18 Britt Kramvik, Margrethe Pettersen, op. cit., p. 140. C'est nous qui traduisons.

19 Raviv Ganchrow, "Long Wave Synthesis ", in Living Earth. Field Notes from the Dark Ecology Project 2014-2016, op. cit., p. 160. 
la topographie et les conditions atmosphériques sur le site. L'installation met en question l'audibilité en tant que facteur délimitant l'environnement. Nous avons souvent tendance à définir les limites de l'environnement en fonction de nos propres perceptions, en excluant aisément un phénomène s'il est inaudible. La question qui se pose est de savoir si d'autres êtres vivants sont capables de percevoir les sons produits par l'installation. La problématique de la pollution sonore n'est pas directement évoquée par l'œuvre, mais il serait intéressant de savoir si d'autres animaux seraient dérangés par ces sons inaudibles pour l'oreille humaine.

Afin de montrer la grande diversité des manifestations organisées dans le cadre du projet " Dark Ecology ", il faut aussi mentionner le travail de Signe Lidén et Espen Sommer Eide. Pendant l'automne 2015, ils ont organisé ce qu'ils ont appelé le Nikel Sound History Club. Pour retracer le passé sonore de la ville, ce club réunissait un groupe de personnes ayant passé une grande partie de leur vie à Nikel. Les organisateurs de ces rencontres ont demandé aux participants de se remémorer des paysages sonores de Nikel à différents moments de l'histoire et de trouver les mots pour décrire leurs souvenirs. L'objectif était de faire revenir les vieux paysages sonores de la ville par l'imagination, et il s'est révélé que les participants étaient capables de se souvenir d'une quantité de sons liés à la vie de tous les jours :

Le bruit quand on fend du bois avant de faire du feu dans le poêle le matin.

Le samovar qui bouillonne. Les clameurs des animaux des fermes collectives.

Le chant d'oiseaux disparus des forêts maintenant brûlées. ${ }^{20}$

L'idée de raconter des histoires en partant de souvenirs sonores n'était pas familière aux participants, mais les organisateurs leur ont proposé de décrocher leur recherche d'une chronologie linéaire pour appliquer une perspective verticale, c'est-à-dire de s'imaginer que les histoires étaient empilées en hauteur. Apparemment, cette consigne a permis de faire des associations surprenantes pour retrouver des moments oubliés. La démarche des artistes est probablement révélatrice de leur vision de l'histoire. Ils semblent la considérer comme constituée d'une multitude de récits qui se superposent au lieu d'être rangés dans un ordre chronologique. Selon les artistes, il faudrait cesser de penser en termes de causalité linéaire pour comprendre les problèmes écologiques auxquels nous sommes confrontés ; tous les paramètres semblent être interconnectés. La notion de mesh développée par Timothy Morton pourrait s'accorder avec leur vision de l'histoire.

20 Espen Sommer Eide, "A Vertical Perspective », in Living Earth. Field Notes from the Dark Ecology Project 2014-2016, op. cit., p. 168. C’est nous qui traduisons. 


\section{Conclusion}

Les artistes appartenant au courant "les arts des nouveaux médias " ont le mérite de proposer une nouvelle approche à l'expérience artistique. En transformant des données perceptuelles par la technologie (en étirant, en inversant, en mettant en boucle, etc.), ils arrivent à donner au public l'impression d'être exposés aux expériences de sources non humaines. Ce changement de perspective pourrait révéler à l'humain que sa perception de l'environnement n'est pas la seule imaginable et que la dichotomie entre la nature et la culture n'a plus de sens. C'est notre tendance à toujours tout ramener à l'échelle humaine et à parler de l'environnement et de la nature au singulier qui est remise en cause.

Le projet "Dark Ecology » a suivi l'exemple de la larve de von Uexküll. Les artistes invités par le projet ont exploré ce que le terrain frontalier entre la Norvège et la Russie cachait à première vue. Ils sont allés au-delà des discours politiques et scientifiques pour porter un autre regard sur les questions écologiques de l'Arctique. Au lieu de décrire l'état des choses, ils ont voulu découvrir des aspects de la réalité qu'on ne voit normalement pas. Leur ambition était de chercher de nouvelles façons de comprendre la crise écologique et de proposer de nouvelles manières de vivre cette époque sombre. Face aux problèmes environnementaux intangibles, le projet a cultivé la poésie et l'esthétique. En dépassant l'espace habituel de la cognition humaine, il a peut-être emmené son public en dehors de sa zone de confort. Mais, en travaillant à la limite des capacités sensorielles humaines, ce projet a permis aux habitants de Kirkenes et Nikel de découvrir l'art comme comme une clé ouvrant aux nouvelles appréhensions de la question écologique. 